\title{
Did a novel virus contribute to late blight epidemics?
}

\author{
Guohong $\mathrm{Cai}^{1^{*}}$, Kevin Myers ${ }^{2}$, William E. Fry ${ }^{2}$ and Bradley I. Hillman ${ }^{3 *}$
}

1. Crop production and Pest Control Research Unit, ARS, USDA and Botany and Plant Pathology Department, Purdue University, West Lafayette, IN

2. School of Integrative Plant Science, Cornell University, Ithaca, NY

3. Department of Plant Biology and Pathology, Rutgers The State University of New Jersey, New Brunswick, NJ

Corresponding authors

E-mail: Guohong.cai@ars.usda.gov (GC)

bradley.hillman@rutgers.edu (BIH) 


\section{Abstract}

$30 \quad$ Phytophthora infestans is the causal agent of potato and tomato late blight. In

31 this study, we characterized a novel RNA virus, Phytophthora infestans RNA virus 2

32 (PiRV-2). The PiRV-2 genome is $11,170 \mathrm{nt}$ and lacks a polyA tail. It contains a single large

33 open reading frame (ORF) with short 5'-and $3^{\prime}$ - untranslated regions. The ORF is

34 predicted to encode a polyprotein of 3710 aa (calculated molecular weight $410.94 \mathrm{kDa}$ ).

35 This virus lacks significant similarity to any other known viruses, even in the conserved

36 RNA-dependent RNA polymerase region. Comparing isogenic strains with or without the

37 virus demonstrated that the virus stimulated sporangia production in $P$. infestans and

38 appeared to enhance its virulence. Transcriptome analysis revealed that it achieved

39 sporulation stimulation likely through down-regulation of ammonium and amino acid

40 intake in $P$. infestans. This virus was faithfully transmitted through asexual reproduction.

41 Survey of PiRV-2 presence in a $P$. infestans collection found it in most strains in the US-8

42 lineage, a very successful clonal lineage of $P$. infestans in North America. We suggest

43 that PiRV-2 may have contributed to its success, raising the intriguing possibility that a

44 potentially hypervirulent virus may contribute to late blight epidemics. 


\section{Author Summary}

47 Potato late blight, the notorious plant disease behind the Irish Potato Famine,

48 continues to pose a serious threat to potato and tomato production worldwide. While

49 most studies on late blight epidemics focuses on pathogen virulence, host resistance,

50 environmental factors and fungicide resistance, we present evidence in this study that a

51 virus infecting the causal agent, Phytophthora infestans, may have played a role. We

52 characterized a novel RNA virus, Phytophthora infestans RNA virus 2 (PiRV-2) and

53 examined its effects on its host. By comparing identical $P$. infestans strains except with

54 or without the virus, we found that PiRV-2 stimulated sporulation of $P$. infestans (a

55 critical factor in late blight epidemics) and increased its virulence. We also profiled gene

56 expression in these strains and identified potential molecular mechanisms through

57 which PiRV-2 asserted its sporulation stimulation effect. In a survey of PiRV-2 presence

58 in a P. infestans collection, we found PiRV-2 in most isolates of the US-8 clonal lineage, a

59 very successful lineage that dominated potato fields in North America for several

60 decades. We suggest that PiRV-2 may have contributed to its success. Our findings raise

61 the intriguing possibility that a potentially hypervirulent virus may contribute to late

62 blight epidemics.

63 


\section{Introduction}

66 Potato late blight caused devastation in the 1840 s and led to food shortage

67 across Europe. In Ireland, where the poor were overwhelmingly dependent on potato, it

68 gave rise to the Irish potato famine - over one million people died and many more were

69 forced to flee [1]. Late blight has proved to be a destructive disease that is difficult to

70 manage and control. Even after extensive research efforts for more than one and a half

71 centuries, it continues to devastate potatoes and tomatoes worldwide and is estimated

72 to cause over 6.7 billion dollars annually in yield losses and control costs [2-4] . Late

73 blight can act very rapidly and leave very little time for growers to suppress it once an

74 epidemic has started.

75 The causal pathogen of potato late blight is named Phytophthora infestans

76 (Mont.) de Bary. It also causes late blight of tomato. P. infestans is a member of

77 oomycetes, which are morphologically similar to filamentous fungi and occupy similar

78 environmental habitats. Historically, P. infestans and other oomycetes were classified as

79 fungi, but they have distinct attributes that separate them from true fungi and in fact

80 are not closely related to fungi. For example, oomycetes have diploid vegetative cells

81 and cellulosic cell walls, while fungi are usually haploid in vegetative state and their cell

82 walls contain chitin. Phylogenetic studies place oomycetes in the major group

83 Stramenopila [5-7], which also includes brown algae and diatoms.

84 By the mid-twentieth century, late blight was managed to tolerable level through

85 various tools, including cultural practices, host resistance, and fungicide usage.

86 However, by the end of twentieth century, late blight came back with vengeance 
87 (reviewed in [8]). The resurgence started in Europe in early 1980s, later in Middle East

88 and Far East, and in North America by late 1980s and early 1990s. The resurgence was

89 caused by multiple independent introductions of exotic and often fungicide-resistant

90 strains from Mexico [9], the center of genetic diversity and origin of $P$. infestans.

91 Prior to the late $20^{\text {th }}$ century, global populations of $P$. infestans outside Mexico

92 were dominated by a single clonal lineage, US-1 (mating type A1) [10]. It was soon

93 replaced world-wide by new strains [11]. In North America, US-8 was arguably the most

94 successful among the introduced lineages. It is extremely virulent on potato, but not as

95 much on tomato [12]. After first being detected in a single county in New York in 1992,

96 it spread to 23 states during 1994 and 1995, and was found in most potato production

97 regions in the United States and part of Canada by 1996 [13]. Many other clonal

98 lineages, up to US-24, have been reported before and after US-8 [14, 15]. Most lineages

99 have come and gone, but US-8 has proved to be enduring.

100 Research on late blight epidemics usually focuses on pathogen virulence, host

101 resistance, environmental factors and fungicide resistance, but any potential role of

102 virus infection of $P$. infestans has not been examined. We previously surveyed $P$.

103 infestans isolates and reported the discovery of four RNA viruses in this organism [16].

104 Three of these viruses have been characterized and their descriptions reported [16-18].

105 Several additional viruses have been reported in other oomycetes [19]. Like many fungal

106 viruses that are asymptomatic in their hosts, these oomycete viruses caused no

107 measurable phenotype in their host organisms. 
110 virus 2 (PiRV-2), a novel RNA virus with a genome of 11,170 nt and no close affinity to

111 other known viruses. This virus stimulated sporangia production and appeared to

112 enhance virulence of $P$. infestans. Transcriptome analysis revealed that PiRV-2 likely

113 stimulated sporulation through restriction of ammonium and amino acid intake. Survey

114 of PiRV-2 presence in P. infestans population suggested that this virus may have

115 contributed to the success of the US-8 lineage and played a role in late blight epidemics.

\section{Results}

\section{Nucleotide sequence analysis of PiRV-2}

Two P. infestans isolates in the US-8 lineage, US940480 and US040009, were

119 previously found to harbor PiRV-2 dsRNA [16]. Repeated effort failed to yield virus-like

120 particles (VLPs) (not shown). DsRNA from US940480 was sequenced by random RT-PCR,

121 RT-PCR with sequence-specific primers, and RNA ligase-mediated RACE (Supplementary

122 Figure S1). After assembly of the overlapping clones, the viral genome was found to be

$12311,170 \mathrm{nt}$, matching previous estimate [16].

124 Examination of the coding potential of all six reading frames revealed a long

125 open reading frame (ORF) on one strand, designated as the positive strand (Fig. 1). This

126 ORF spanned from nt 7 to 11,139 , and could encode a polyprotein of 3710 aa (calculated

127 molecular weight $410.94 \mathrm{kDa}$ ). The first AUG codon in the ORF at nt 7-9 had an A at both

$128-3$ and +4 positions, predicted to be a more favorable context than the two AUG codons

129 immediately downstream in this reading frame (nt 253-255 and 292-294, respectively) 
$130[20,21]$, both of which had a pyrimidine (C) at -3 position. Pending experimental

131 evidence, we assumed for sequence analysis purposes the AUG codon at nt 7-9 was the

132 translation initiation site. This implied a short $5^{\prime}$ untranslated region (UTR) of 6 nt and a

133 3' UTR of $31 \mathrm{nt}$. A signal peptide was not identified at the $\mathrm{N}$-terminal region of this

134 protein, but 14 potential transmembrane regions in two clusters were predicted - one

135 in the middle section and one close to the C-terminus (Fig 1). No other significant ORFs

136 were found on either strand of PiRV-2/US940480.

PiRV-2 dsRNA from $P$. infestans isolate US040009 was also sequenced

138 (Supplementary Figure S1). PiRV-2/US940480 and PiRV-2/US040009 shared 99.5\%

139 nucleotide identity, with 58 single nucleotide polymorphisms (SNPs) between the two

140 sequences; there were no indels. There were 25 synonymous SNPs while the other 33

141 were non-synonymous SNPs, and no SNP induced premature termination of the ORF.

142 All downstream analysis was based on PiRV-2/US940480.

\section{PiRV-2 dsRNA encodes a putative viral RNA-dependent RNA polymerase but lacks}

144 similarity to any known viruses

145 At the nucleic acid level, no significant similarity was found between PiRV-2 and

146 any other sequences in GenBank non-redundant database. When the deduced protein

147 sequence was used as query in BLASTP searches, only marginal similarities were

148 identified between part of PiRV-2-encoded protein and the RNA-dependent RNA

149 polymerase (RdRp) regions of several astroviruses (e.g. Mamastrovirus 3, GenBank

150 accession ALA16028, bit score 50.1, E-value 0.15). RdRps of astroviruses contain RdRp_1

151 domain (pfam00680). Searches against various protein domain profile databases 
152 identified an RdRp domain close to the C-terminus of the PiRV-2-encoded protein. For

153 example, in a search of the Conserved Domain Database v3.16 [22], an RdRp (cd01699)

154 was identified at aa 3264-3407 (E-value 3.13e-03). Similarly, scanning Prosite (release

155 20.120), a database of protein domains, families and functional sites as well as

156 associated patterns and profiles [23, 24], identified PS50507 (RdRp of positive ssRNA

157 virus catalytic domain profile) at aa 3262 - 3392 (score10.724). In contrast, a search of

158 the Pfam database did not find significant similarity between PiRV-2 and any RdRps or

159 other protein domains/families.

160

All RdRps and many DNA-directed polymerases employ a right-hand shape fold

161 with three subdomains termed fingers, palm and thumb [25]. Only the palm subdomain

162 is well conserved among all of these enzymes. In RdRps, the palm subdomain comprises

163 three well conserved motifs, D-x(4,5)-D, [S, T]-G-x3-T-x3-N and G-D-D [25, 26], where

164 entries in the bracket represent amino acid options in that position, $\mathrm{x}$ stands for any

165 amino acid, and numbers represent the number of preceding amino acids. An exception

166 is the virus family Birnaviridae, which lacks the G-D-D motif [27]. All three motifs were

167 found in PiRV-2 (Fig 2A). Based on the above data, we concluded that PiRV-2 encoded

168 an RdRp. An alignment of core RdRp regions from representative viruses with RdRP_1 or

169 RdRP_4 domain was built (Fig 2A), and a neighbor joining tree based on this alignment

170 showed that PiRV-2 did not fall into any known virus families (Fig 2B).

171 In addition to the RdRp region, scanning the Prosite database also found the

172 motif signature of eukaryotic thiol (cysteine) proteases histidine active site (PS00639) at

173 aa 744-754 (SYHSVLMAGCS). Eukaryotic thiol proteases have an essential catalytic triad 
174 of cysteine, histidine and asparagine. The motif signatures of the cysteine and

175 asparagine active sites were not found, either by scanning Prosite database or by visual

176 inspection. It remains to be determined whether PiRV-2 encodes a protease around the

177 identified histidine active site.

178 PiRV-2 stimulates sporangia production in $\boldsymbol{P}$. infestans and enhances its virulence

179 To examine the effect of PiRV-2 on its host, the virus was cured from isolate

180 US940480 and then re-introduced into the cured isolates through anastomosis. To cure

181 PiRV-2, US940480 was grown on Ribavirin-containing rye agar and transferred by

182 hyphae-tipping. After four iterations, the virus was found to be cured in two cultures, as

183 confirmed by dsRNA extraction and RT-PCR (Supplementary Figure S2). These two

184 cultures were named US940480/PiRV-2C1 and US940480/PiRV-2C2, respectively. To

185 introduce PiRV-2 back into cured isolates, US940480 was co-cultured in close proximity

186 with US940480/PiRV-2C1 or US940480/PiRV-2C2 on rye agar and an agar plug was taken

187 from the side of cured isolates. Virus transmission was confirmed by RT-PCR

188 (Supplementary Figure S3). The resultant cultures were named US940480/PiRV-2T1 and

189 US940480/PiRV-2T2, respectively.

US940480, US940480/PiRV-2C1, US940480/PiRV-2C2, US940480/PiRV-2T1 and

191 US940480/PiRV-2T2 were grown on rye agar to compare their growth rate, colony

192 morphology and sporulation. Slightly but significantly $(P=0.05)$ faster growth rate was

193 observed in the virus-cured isolates. The virus-cured isolates also produced denser

194 aerial mycelium compared to US940480 based on visual observation (Fig. 3A). The most

195 profound difference was observed in sporangia production: virus-infected isolate 
196 US940480 produced abundant sporangia, while cured isolates US940480/PiRV-2C1 and

197 US940480/PiRV-2C2 sporulated sparsely (Fig. 3A, inset). On average, the PiRV-2-

198 containing isolate US940480 produced 9 to 125 times as many sporangia as its isogenic,

199 virus-free counterparts US940480/PiRV-2C1 and US940480/PiRV-2C2 did, and the

200 difference was always statistically significant $(P=0.05$, Fig $3 \mathrm{C}$ and not shown).

201 Co-culturing of US940480 with US940480/PiRV-2C1 or US940480/PiRV-2C2 re-

202 introduced the virus into the virus free isolates (Supplementary Figure S3 and not

203 shown) and restored their sporangia production (Fig. 3B-C). Sporangia production in

204 US940480/PiRV-2T1 and US940480/PiRV-2T2 were not significantly different from

205 sporangia production in US940480 (Fig. 3C).

206 To determine the impact of PiRV-2 on host virulence, US940480 and

207 US940480/PiRV-2C1 were used to infect leaves of susceptible potato cultivar Red La

208 Soda in detached leave assay. Lesions caused by US940480 were significantly larger than

209 those caused by US940480/PiRV-2C1 (Fig. 3D). We were not able to obtain sufficient

210 numbers of sporangia from US940480/PiRV-2C2 to compare those from US940480.

211 PiRV-2 causes transcriptome changes in $\boldsymbol{P}$. infestans

212 To understand the molecular mechanism(s) through which PiRV-2 acted on its

213 host, transcriptomes of US940480 and US940480/PiRV-2C1 were sequenced. Three

214 independent mRNA libraries each for US940480 and US940480/PiRV-2C1 were

215 sequenced on Illumina Miseq platform, generating 5.08 million to 7.04 million reads for

216 each library. The reads were first mapped to the PiRV-2 genome. Two out of three

217 US940480 mRNA libraries contained one and two reads, respectively, that were aligned 
218 to PiRV-2. No read from the three US940480/PiRV-2C1 mRNA libraries was aligned to

219 the virus genome. This was in agreement with the fact that US940480 harbored PiRV-2

220 while US940480/PiRV-2C1 did not. Since PiRV-2 did not have a polyA tail, and mRNA was

221 extracted from total RNA using polyT beads, only trace amount of PiRV-2 RNA was

222 introduced into the libraries.

223 The sequence reads were then mapped to the genome of $P$. infestans [28] to

224 obtain read counts for individual genes. With a Benjamini-Hochberg adjusted $P$ value of

2250.01 and a minimum of $\log _{2}$ fold change (LFC) of 1 (2-fold change) requirements, 848

226 differentially expressed genes (DEGs) were identified, with 431 genes up-regulated by

227 PiRV-2 and 417 genes down-regulated by this virus (Supplementary Table S3).

$228 \quad$ Four groups of genes were enriched in up-regulated DEGs. The first group (i)

229 was histone protein genes. Of the 18 histone protein genes annotated in P. infestans

230 genome, 9 were up-regulated, a 20.6-fold enrichment. Two additional histone genes

231 were induced to a lesser extent (padj $<0.05$, LFC $>0.5$ ) and none was significantly down-

232 regulated (Supplementary Figure S4A and Supplementary Table S4). Also enriched were

233 (ii) genes for flagellum-related proteins, (iii) proteins with epidermal growth factor

234 (EGF)-like conserved site, and (iv) genes in the glycolytic process (Supplementary Figure

235 S4 B, C and D), with 10.0-, 9.9- and 7.4-fold enrichment, respectively.

236 Genes in the ribosome pathway were highly represented in the down-

237 regulated DEGs. Of the 110 genes in $P$. infestans that mapped to the KEGG ribosome

238 pathway, 23 were down-regulated, a 9-fold enrichment. An additional 34 genes in this

239 pathway were down-regulated to a lesser extent (padj $<0.05$, LFC $<-0.5$ ) and no gene in 
240 this pathway was significantly up-regulated (Supplementary Table S5 and

241 Supplementary Figure S5). These down-regulated genes included both the large and

242 small subunit ribosome proteins. The down-regulated DEGs also included a group of 98

243 genes with transmembrane domain (Supplementary Table S6), but with only 1.5-fold

244 enrichment. These genes included many involved in nutrient transport across the

245 membrane, as well as several kinases and other genes. The potential molecular

246 pathway(s) PiRV-2 employed to act on P. infestans were further explored in the

247 "Discussion" section.

248 Distribution of PiRV-2 in P. infestans

249 Since PiRV-2 stimulated sporulation in P. infestans and enhanced its virulence,

250 we suspected that it could play a role in late blight epidemics and set out to examine its

251 presence in $P$. infestans population. In a previous study, we screened 22 isolates of $P$.

252 infestans using dsRNA extraction [16]. In the current study, we screened 54 isolates for

253 PiRV-2 using RT-PCR (Fig 4 and not shown). The combined results are detailed in

254 supplementary Table S1 and summarized in Table 1. In North America, the virus was

255 detected in 11 out of 13 isolates in the US-8 lineage and 3 out of 4 isolates in US-22

256 lineages, but not in 6 isolates belonging to US-11, US-17, US-23 or isolates whose

257 lineages were not determined. From isolates collected outside of the USA, PiRV-2 was

258 not detected in 9 isolates from Estonia, The Netherlands and South America. However,

259 PiRV-2 was detected in 1 out of 41 isolates from Mexico. 
263 Table 1. PiRV-2 distribution in Phytophthora infestans

\begin{tabular}{|l|l|c|c|c|}
\hline \multicolumn{2}{|c|}{ Location/lineage } & $\begin{array}{c}\text { \# of } \\
\text { isolates }\end{array}$ & PiRV-2 positive & $\begin{array}{c}\text { PiRV-2 } \\
\text { negative }\end{array}$ \\
\hline USA & US-8 & 13 & 11 & 2 \\
\hline & US-17 & 1 & 0 & 1 \\
\hline & US-11 & 1 & 0 & 1 \\
\hline & US-22 & 4 & 3 & 1 \\
\hline & US-23 & 1 & 0 & 1 \\
\hline & ND & 3 & 0 & 3 \\
\hline South Africa & & 1 & 0 & 1 \\
\hline The Netherlands & & 5 & 0 & 5 \\
\hline Estonia & 3 & 0 & 3 \\
\hline Mexico & 41 & 1 & 40 \\
\hline
\end{tabular}

264

265 PiRV-2 is faithfully transmitted through asexual reproduction

To determine PiRV-2 transmission through asexual reproduction, single-

267 sporangia cultures were obtained from isolate US940480. RT-PCR was used to assay

268 PIRV-2 in 29 single-sporangia cultures, and the virus was detected in all of them (Fig. 5).

\section{Discussion}

In this study, we characterized PiRV-2, a novel RNA virus, in the potato and

271 tomato late blight pathogen, $P$. infestans. Efforts to purify VLPs were not successful, but

272 this was not surprising. It is not uncommon for viruses infecting the lower eukaryotes

273 such as oomycetes and fungi to lack protein capsids since most do not infect through an

274 extracellular route. Whether the PiRV-2 dsRNA constitutes the "virus genome" or the

275 replicative form of a single-stranded RNA virus is a matter of definition: viruses in the 
276 fungus-infecting family Hypoviridae have no capsid protein, but their replicative form

277 dsRNA is encapsulated with an RdRp complex in lipid vesicles [29]. Genomes of these

278 viruses are organizationally and phylogenetically most similar to positive-sense RNA

279 viruses; therefore some virologists consider them to be ssRNA viruses while others

280 consider them to be dsRNA viruses because of its encapsulation in vesicles. Although the

281 RdRp region of PiRV-2 showed some similarity to RdRp_1 domain, which suggests a

282 single-strand RNA genome, the similarity was only marginal. In fact, the RdRp in PiRV-2

283 was only identified by looking for the three well-conserved motifs in the palm domain of

284 RdRp. Both the 5' UTR (GUUAAA, 83\% AU) and the 3' UTR

285 (AUUAAGAUGCAUAAUUAAACACAACUUAAGC, 74\% AU) of PiRV-2 were AU-rich while

286 the complete sequence had a 52\% AU content. An AU-rich 5' UTR on the plus strand is a

287 general feature of dsRNA viruses and is the region where the dsRNA separates during

288 virus replication as the polymerase enters [30]. No other similarity was observed

289 between PiRV-2 and previously reported viruses. Phylogenic analysis confirmed that

290 PiRV-2 does not belong to any known virus families or groups. We conclude that PiRV-2

291 belongs to a new virus family yet to be described.

292 Many viruses of lower eukaryotes are asymptomatic or cause only mild

293 symptoms in their hosts, but others cause observable symptoms [19, 31, 32]. In

294 phytopathogenic fungi, a small number of viruses can attenuate the virulence of their

295 hosts, resulting in a phenomenon called hypovirulence. The best example of

296 hypovirulence was illustrated by Cryphonectria hypovirus 1 [33]. L1 dsRNA in Nectria

297 radicicola, the causal agent of ginseng root rot, showed the opposite effect. This virus 
298 stimulated sporulation and increased virulence of N. radicicola [34]. Alternaria alternata

299 chrysovirus 1 (AaCV1) showed two-sided effects. AaCV1 was isolated from Alternaria

300 alternate Japanese pear pathotype, which infects the susceptible pear cultivar

301 'Nijisseiki', produces AK-toxin and causes Alternaria black spot on the leaves. On the one

302 hand, high titer of AaCV1 severely impaired the growth of A. alternata; on the other

303 hand, it increased AK-toxin production and enhanced virulence of this fungus [35].

304 In this study, PiRV-2 appears to have shown similar effects to its host as L1

305 dsRNA in N. radicicola, in that it stimulates sporangium production and enhances

306 virulence. The sporangium plays an important role in the late blight disease cycle [36].

307 Sporangia are dispersed via air or water to healthy tissue to initiate new infections.

308 Within a few days of inoculation, the pathogen produces a macroscopically visible lesion

309 from which many sporangia can be produced and each is capable of initiating another

310 cycle of pathogenesis.

311 Given the effects of PiRV-2 on P. infestans, it's reasonable to suspect that this

312 virus could play a role in late blight epidemics. Survey of $P$. infestans collection showed

313 that this virus was present in most isolates of the US-8 clonal lineage of this pathogen.

314 The US-8 lineage was first detected in the United States in 1992 and soon spread to

315 most potato production areas in North America [13]. From the mid-1990s until 2009,

316 US-8 had been the dominant lineage in the USA. Recently (2017), US-23 has been

317 dominant, but US-8 still constituted a substantial portion of $P$. infestans isolated from

318 potato in 2016 and 2017 [4]. The virulence of $P$. infestans depends on many factors,

319 such as inoculum production, temperature adaptation and host preference [15]. We 
320 suggest that PiRV-2 may have been a contributing factor to the success of the US-8

321 lineage. PiRV-2 was found in one of 46 isolates from Mexico. It is assumed that the US-7

322 and US-8 lineages migrated to the United States in 1992 through the trade of infected

323 plant material [37]. Likely one of the strains carried by the infected material harbored

324 PiRV-2 and led to the dominance of US-8 for several decades in many potato fields in

325 North America.

326 Clonal lineage US-22 was detected in 2007 and US-23 and US-24 were reported

327 in 2009 [14]. The late blight epidemics in 2009 was mostly caused by US-22. Infected

328 tomato transplants infected by US-22 were distributed by a single supplier and sold by

329 major distributors to many households. It caused wide-spread infection in garden

330 tomatoes, which then spread to commercial tomato and potato fields [12]. Three out of

331 four US-22 isolates examined in this study contained PiRV-2. Further population and

332 epidemiology studies are needed to understand the role of PiRV-2 in late blight

333 epidemics.

334 At the transcriptome level, the strain harboring PiRV-2 had significantly lower

335 expression of many ribosome protein genes comparing to its isogenic, virus-free

336 counterpart. The ribosome is the core of protein translation machinery. In Escherichia

337 coli, Neurospora crassa and Saccharomyces cerevisiae, the expression of ribosome

338 protein genes is proportional to growth rate and down-regulated by nutrient limitations

339 [38-40]. In the diploid yeast S. cerevisiae, nitrogen starvation induces sporulation and

340 down-regulation of more than 40 ribosome protein genes. When nitrogen is

341 replenished, these genes return to normal expression level and the yeast resumes 
342 vegetative growth $[41,42]$. While the sporulation in S. cerevisiae is a sexual process,

343 resulting in haploid progenies from diploid parent, nitrogen starvation also induces

344 asexual conidiation in fungi $N$. crassa and Aspergillus nidulans $[43,44]$. There are five

345 annotated ammonium transporter genes in $P$. infestans. Among these, the three genes

346 with the highest expression level were significantly down-regulated by PiRV-2

347 (Supplementary Table S7). In yeast, down-regulation of ribosome protein genes can also

348 be induced through amino acid deprivation [45]. Among the 34 amino acid permease

349 genes in P. infestans, eight were significantly down-regulated by PiRV-2 and none was

350 up-regulated (Supplementary Table S7).

351 The main function of histone proteins in eukaryotic cells is to package and

352 order DNA. When S. cerevisiae is induced into sporulation through nitrogen starvation,

353 the amount of histone proteins per cell increases many folds in the early hours, followed

354 by increase of DNA synthesis [46]. The four major families of histones, H2A, H2B, H3 and

$355 \mathrm{H}$ 4, form the nucleosome core on which DNA wraps around, while $\mathrm{H} 1$ links individual

356 nucleosome together to form higher order structure. Multiple histone protein genes

357 were highly induced by PiRV-2, including all five major histone families (Supplementary

358 Figure S4A and Supplementary Table S4).

359 In our study, none of the DNA polymerase genes was significantly affected by

360 PiRV-2 but DNA content was not quantified. Genes in one of the up-regulated groups,

361 the seven genes with EGF-like domain, have functions predicted to affect DNA synthesis

362 (Supplementary Figure S4B). EGF-like domains are usually found in the extracellular

363 portion of membrane proteins or in proteins that are secreted. Five of the seven up- 
364 regulated genes were predicted to encode extracellular proteins. In animal cells, binding

365 of an EGF-like domain to a cell surface receptor is essential for activation of tyrosine

366 kinase in the receptor cytoplasmic domain, which initiates a signal transduction and

367 results in DNA synthesis and cell proliferation $[47,48]$.

368 Previous studies have identified two genes with regulatory functions that are

369 critical for sporangium production in P. infestans. A G protein $\beta$-subunit (Pigpb1) is

370 induced in nutrient starved medium prior to the onset of sporangium formation, and

371 silencing of this gene results in drastic decrease in sporangia production and dense

372 growth of aerial mycelium [49]. Picdc14 is a cell-cycle phosphatase and expressed only

373 in sporangiophore initials, sporangiophore and sporangia[50]. Silencing of Picdc14 also

374 impairs sporangium production. Picdc14 is also induced by nutrient starvation and it is

375 assumed to act after Pigpb1 because silencing of the latter down-regulates its

376 expression $[49,51]$. In our study, Pigpb1 (PITG_06376) was not significantly affected by

377 PiRV-2. The expression of Picdc4 (PITG_18578) was up-regulated by 4-fold by PiRV-2 but

378 there was high variability among the replicates $(\mathrm{LFC}=1.97, P=0.048, \mathrm{padj}=0.215)$.

379 The analysis above suggests a potential molecular mechanism for stimulation

380 of sporulation in P. infestans by PiRV-2 (Fig. 6). The virus, directly or indirectly, down-

381 regulates the expression of ammonium and amino acid transporter genes, resulting in

382 nitrogen and amino acid deprivation. This leads to decrease in many ribosome proteins

383 and increase of histone proteins, primes $P$. infestans for DNA synthesis through up-

384 regulation of genes with an EGF-like domain, and possibly up-regulates the expression

385 of Picdc14. These molecular processes result in less vegetative growth in strains 
386 containing PiRV-2 (sparse aerial mycelium) and more cell proliferation (increased

387 sporangium production) in those strains.

\section{Materials and Methods}

389 Cultures of $\boldsymbol{P}$. infestans. $P$. infestans isolates used in this study and their relevant

390 information are listed in Supplementary Table S1. The isolates were maintained on rye agar.

391 For mycelium production, the isolates were grown in pea broth at $18{ }^{\circ} \mathrm{C}$ in darkness.

392 RNA extraction. DsRNA was extracted from approximately two-week old mycelium with CF-

39311 cellulose following the method of Morris and Dodds [52] modified by Tooley et al [53].

394 DNasel and S1 nuclease were used as previously described [16] to remove traces of

395 genomic DNA and ssRNA, respectively. Total RNA was extracted from mycelium using

396 RNeasy Plant Mini Kit (Qiagen) according to manufacturer's instructions.

397 Primers. Primers and adapters used throughout this study are listed and described in

398 Supplementary Table S2.

399 cDNA cloning, sequencing, and sequence analysis. PiRV-2 was previously found in two

400 isolates of $P$. infestans, US940480 and US040009. The dsRNA from isolate US940480 was

401 used as template for PiRV-2 sequencing. A random cDNA library was constructed as

402 previously described [16], and their sequences were assembled into contigs. Sequence-

403 specific primers were then used in various combinations in reverse transcription (RT)-

404 PCR to confirm sequences of these contigs and to link the contigs in correct order. Three

405 independent rounds of RT-PCRs were performed. The primers were designed based on

406 sequences of random clones and sequences of clones from previous round(s) of RT-PCR. 
407 To obtain terminal sequences, a 5'-phosphorylated and 3'-blocked adapter $\left(5^{\prime} \mathrm{PO}_{4}{ }^{-}\right.$

408 AGGTCTCGTAGACCGTGCACC-NH2-3') was ligated to the 3' end of each strand of the

409 dsRNA using T4 RNA ligase (Promega) [54, 55]. Primer Padapter (5'-

410 GGTGCACGGTCTACGAGACCT-3'), reverse complement to the adapter, was used in

411 combination with sequence-specific primers to amplify terminal regions (Supplementary

412 Figure S1). Superscript III reverse transcriptase and Platinum Taq high fidelity enzyme

413 mix (Invitrogen) were used for RT-PCR following manufacturer's instructions. Amplified

414 fragments were cloned into PCR2.1-TOPO vector (Invitrogen). One or more clones from

415 each RT-PCR were sequenced. All sequencing was conducted using Big Dye Terminator

416 chemistry. Sequences were assembled using Sequencher (Gene Codes Corp.). After

417 PiRV-2/US940480 was sequenced, PiRV-2/US040009 was amplified into two overlapping

418 RT-PCR fragments, and they were purified and sequenced directly (Supplementary

419 Figure S1). The virus genome sequences were deposited in GenBank (accession \#s:

$420 \mathrm{MH013270}$ and MH013271).

421 ORF Finder (http://www.ncbi.nlm.nih.gov/gorf/gorf.html) was used to identify

422 potential ORFs using standard genetic codes. The nucleic acid and deduced protein

423 sequences of PiRV-2 were used to search various databases as described in the "Results"

424 section using default settings. Identification of potential signal peptide and

425 transmembrane regions were carried out using SignalP 4.1 server [56] and TMHMM

426 server v.2.0 [57], respectively. Sequence alignment of RdRps was conducted using the

427 Muscle program in MEGA v.6.0 [58] with visual inspection, and a neighbor-joining tree

428 was constructed and bootstrapped with 1000 replicates. 
429 PiRV-2 curing. Isolate US940480 was grown on a 9-cm diameter rye agar plate

430 containing $50 \mathrm{mg} / \mathrm{ml}$ Ribavirin (SigmaAldrich) at $18^{\circ} \mathrm{C}$ in the dark. When the colony

431 diameter was approximately two-thirds of the diameter of the plate, a hyphal tip was

432 taken from the edge with the aid of a dissecting microscope and transferred to a new

433 plate. This process was repeated and PiRV-2 curing in the hyphae-tipping cultures was

434 periodically examined using dsRNA extraction and RT-PCR. For this purpose, the hyphae-

435 tipping cultures were grown in pea broth without Ribavirin to produce mycelium. DsRNA

436 and total RNA were extracted from the mycelium as described above. dsRNA samples

437 were run on $1 \%$ agarose gel, stained with ethidium bromide and photographed under uv

438 light. SuperScript III one-step RT-PCR system (Invitrogen) was used in RT-PCR, with total

439 RNA as template and primer pairs P05/P07 or P24/P27 (supplementary Table S2) in

440 separate reactions and 40 amplification cycles. The RT-PCR products were separated on

441 agarose gels, stained and photographed as described above. The hyphal-tipping cultures

442 derived from US940480 in which PiRV-2 was cured were named US940480/PiRV-2C1

443 and US940480/PiRV-2C2.

444 PiRV-2 transmission through anastomosis. Agar plug from the edge of actively growing

445 US940480 was placed in close proximity with an agar plug from US940480/PiRV-2C1 or

446 US940480/PiRV-2C2 on rye agar plates and grown at $18{ }^{\circ} \mathrm{C}$ in the dark. After two weeks,

447 an agar plug was taken from the side of PiRV-2-cured cultures and placed on a fresh rye

448 agar plate. RT-PCR was used to detect PiRV-2. The resultant cultures from

449 US940480/PiRV-2C1 and US940480/PiRV-2C2 into which PiRV-2 was re-introduced were

450 named US940480/PiRV-2T1 and US940480/PiRV-2T2, respectively. 
451 Phenotype of PiRV-2. Agar plugs from US940480, US940480/PiRV-2C1, US940480/PiRV-

452 2C2, US940480/PiRV-2T1 and US940480/PiRV-2T2 were taken from the edges of actively

453 growing cultures with a \#5 cork borer and transferred to the center of fresh 9-cm

454 diameter rye agar plates. The plates were incubated at $18{ }^{\circ} \mathrm{C}$ in the dark. Colony

455 diameter was measured at day 5, 7 and 9 after inoculation by averaging two

456 measurements at 90 degree difference. Two weeks after incubation, when the plates

457 were fully covered, they were photographed. Sporangia were harvested by flooding the

458 colonies with $10 \mathrm{ml}$ distilled water and rubbing with a sterile glass rod, and

459 concentrated to $2 \mathrm{ml}$ by low-speed centrifugation. Sporangia concentration was

460 determined using a hemocytometer. The experiment was conducted twice with three

461 plates per isolate in each experiment.

462 Pathogenicity test. Sporangia were harvested from 2-week old cultures of US940480

463 and US940480/PiRV-2C1 as described above. Sporangia concentration was adjusted to

$4643.5 \times 10^{5}$ per $\mathrm{ml}$ with the aid of a hemocytometer. Leaves were excised from greenhouse-

465 grown Red La Soda potato plants near flowering stage. A 50 ul aliquot of sporangia

466 suspension (17,500 sporangia) was placed in the center of individual leaves. Control

467 leaves were inoculated with sterile $\mathrm{H}_{2} \mathrm{O}$. Three leaves were used per treatment. The

468 inoculated leaves were placed in a moisture chamber and incubated in a growth

469 chamber at $16{ }^{\circ} \mathrm{C}$ and 12 -hr light. Lesion diameter was measured by averaging two

470 measurements at 90 degree difference after 7 days.

471 Transcriptome sequencing and analysis. Agar plugs from the edge of actively growing

472 cultures of isolates US940480 and US940480/PiRV-2C1 were transferred to rye agar 
473 plates overlaid with cellophane, with three plates per isolate. After incubation at $18{ }^{\circ} \mathrm{C}$

474 in dark for 1 week, mycelium from each plate was harvested and total RNA was

475 extracted using RNeasy Plant Mini Kit (Qiagen). An Illumina TruSeq mRNA library was

476 made for each sample according to manufacturer's instructions. The six libraries, each

477 with distinct barcode, were pooled and sequenced on Illumina Miseq platform for two

478 runs, with the first run at $1 \times 65 \mathrm{bp}$ and the second run at $1 \times 66 \mathrm{bp}$.

479 Read quality was examined using FastQC [59]. The adapter sequences were

480 trimmed using fastx_clipper program in the FASTX-Toolkit [60]. Reads less than 25 bp

481 after trimming were removed from downstream analysis. The sequence reads were first

482 mapped to PiRV-2 using TopHat v2.1.0 [61] and Bowtie2 v2.3.3.1 [62] to determine if

483 the libraries contained PiRV-2-derived sequences. The genome assembly and

484 annotation of $P$. infestans strain T30-4 [28] were downloaded from GenBank (assembly

485 ASM14294v1). The sequence reads were then mapped to the genome of $P$. infestans

486 and the read counts that mapped to individual protein-coding genes were obtained

487 using HTSeq-count [63]. Differentially expressed genes were analyzed using DESeq2

488 [64]. Functional analysis of differentially expressed genes were conducted using DAVID

489 version 6.8 [65] with default settings. The transcriptome data were submitted to

490 GenBank (BioProject accession \#: PRJNA437643).

491 Detection of PiRV-2 in P. infestans population. A panel of 54 isolates was maintained

492 on rye agar. They were transferred to pea broth for mycelium growth. Total RNA was

493 extracted from mycelium. Two RT-PCR reactions, with primer pairs $\mathrm{p} 16 / \mathrm{p} 18$ and

$494 \mathrm{p} 24 / \mathrm{p} 27$, respectively, were used to detect PiRV-2 in each isolate. 
495 PiRV-2 transmission through sporangia. Sporangia were harvested from 2-week old

496 cultures of US940480 as described above. The concentration of the sporangia

497 suspension was adjusted to 1,000 sporangia/ml with the aid of a hemocytometer. A

498 rye agar plate was inoculated with $0.3 \mathrm{ml}$ sporangia suspension, spread with a sterile

499 glass rod, air-dried under a hood, and incubated at $18^{\circ} \mathrm{C}$ in the dark. Germinating single

500 sporangia were transferred to fresh rye agar plates with the aid of a dissecting

501 microscope to obtain single-sporangium cultures of US940480. To determine the

502 presence of PiRV-2, single-sporangia cultures were grown in pea broth and total RNA

503 was extracted from mycelium. RT-PCR with primer pair p16/p18 was used to detect

504 PiRV-2.

505

507 the purpose of providing specific information and does not imply recommendation or

508 endorsement by the U.S. Department of Agriculture. USDA is an equal opportunity

509 provider and employer. 


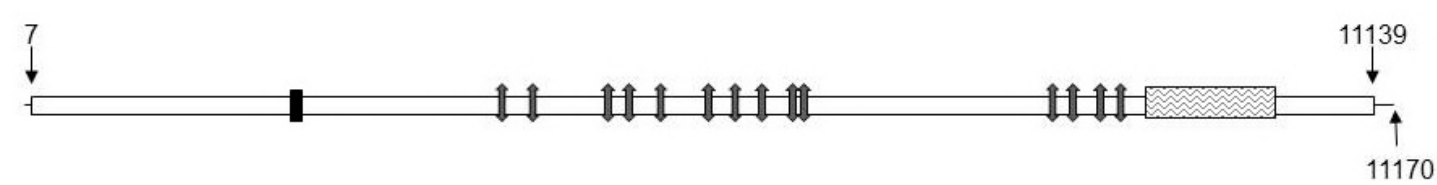

目 RNA-dependent RNA polymerase

Thiol (cysteine) proteases histidine active site

\Transmembrane region

519 Fig. 1. Genome structure of PiRV-2. Lines represent the 5' and 3' untranslated regions,

520 the open box represents the predicted single ORF of $11,130 \mathrm{nt}$ beginning at $\mathrm{nt} 7$ and

521 terminating at nt 11,139 , and the numbers indicate nucleotide positions. The few

522 predicted functional domains identified on the 3,710 aa protein sequence are provided. 
bioRxiv preprint doi: https://doi.org/10.1101/383653; this version posted August 2, 2018. The copyright holder for this preprint (which was not certified by peer review) is the author/funder. This article is a US Government work. It is not subject to copyright under 17 USC 105 and is also made available for use under a CCO license.

A

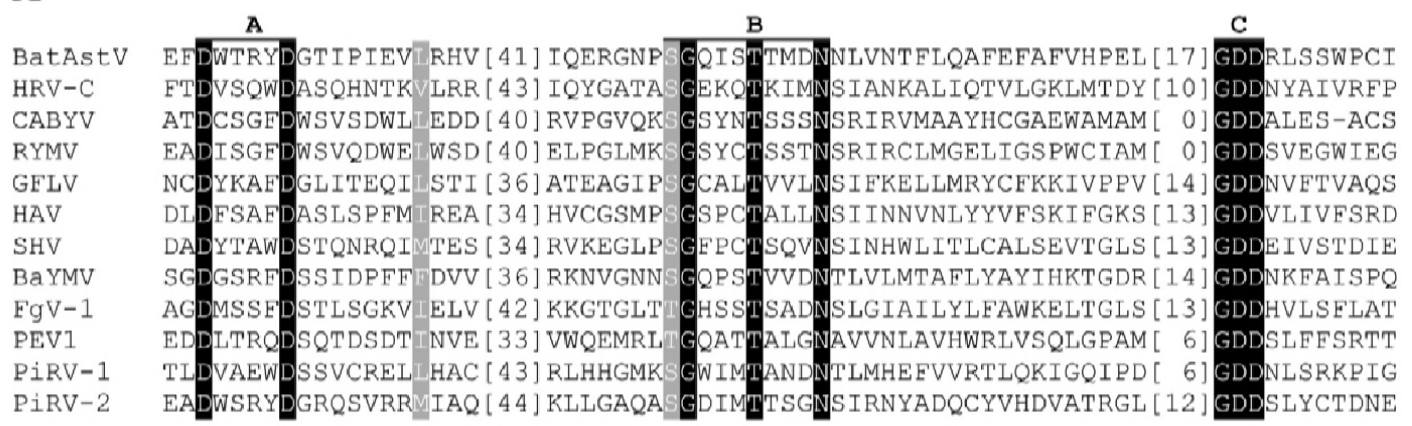

B

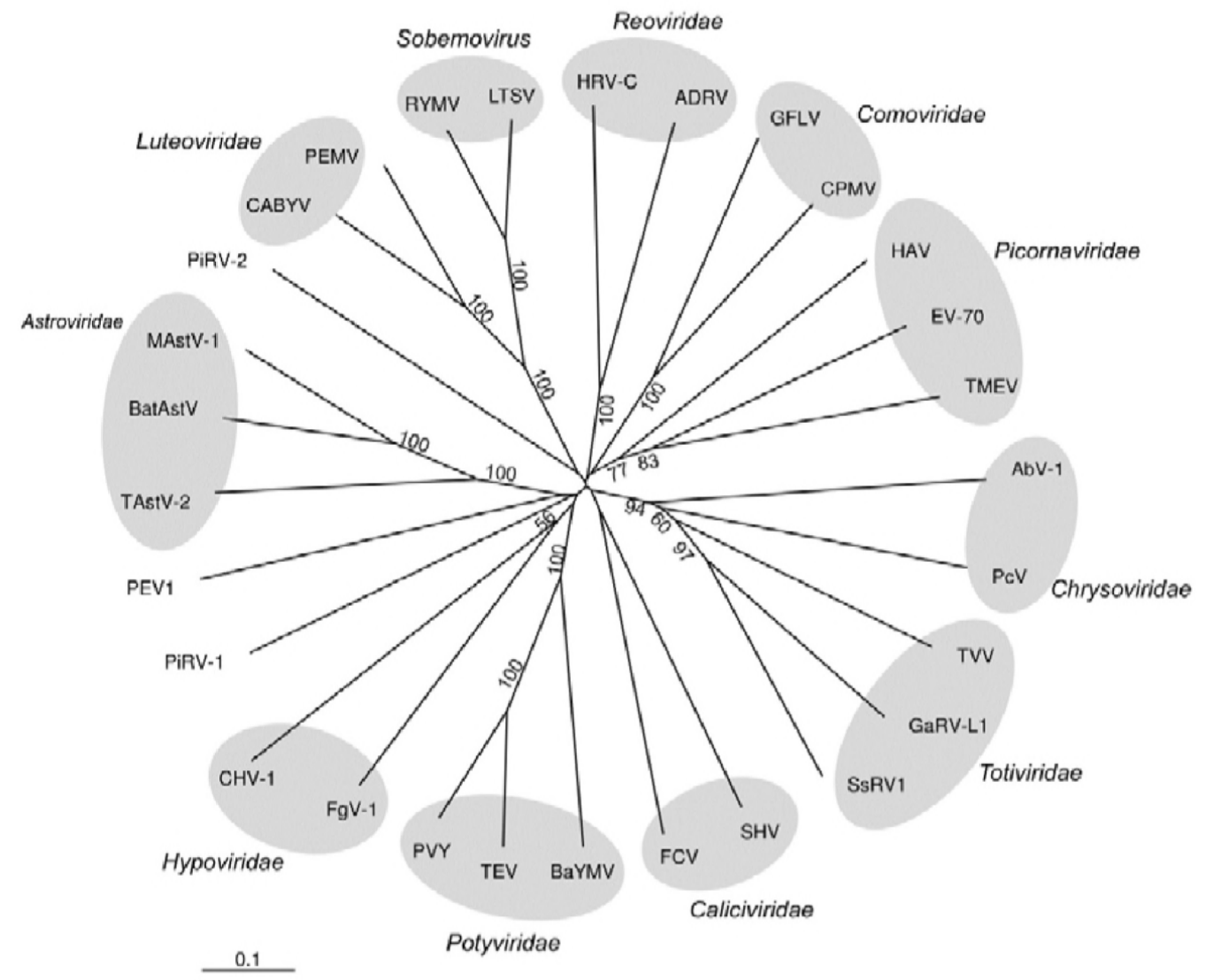

526 Fig. 2. PiRV-2 encodes a predicted viral RNA-dependent RNA polymerase with no close

527 similarity to known viruses. A. A subset of the alignment of RdRp regions of PiRV-2 and

528 other RNA viruses, including ssRNA and dsRNA viruses. Three conserved motifs of RdRp

529 palm subdomain, A-C, are labeled on the top. Amino acid residues identical in all viruses

530 are highlighted with a black background and white lettering; those belonging to the 
531 same group are highlighted with a gray background and white lettering. The grouping of

532 amino acids based on similarity is as follows: $A, G ; L, I, V, M, F, Y, W ; S, T ; K, R ; D, E, N, Q$.

533 Numbers within the brackets indicate the number of amino acids not shown. $B$.

534 Neighbor-joining tree based on the alignment of RdRps between motifs $A$ and C.

535 Representatives from diverse virus groups were included. A subset of the alignment is

536 shown in A. Bootstrap values (1000 replicates) in percentage are labeled for branches

537 with more than $50 \%$ support. Full virus names and EMBL/GenBank/DDBJ accession

538 numbers for the protein sequences (in parentheses) are: AbV-1, Agaricus bisporus virus

5391 (Q90193); ADRV, Adult diarrhea rotavirus (P35942); BatAstV, Bat astrovirus

540 (ACF75855); BaYMV, Barley yellow mosaic virus (Q04574); CABYV, Cucurbit aphid-borne

541 yellows virus (Q65969); CHV-1, Cryphonectria hypovirus 1 (AAA67458); CPMV, Cowpea

542 mosaic virus (P03600); EV-70, Human enterovirus 70 (P32537); FCV, Feline calicivirus

543 (P27407); FgV-1, Fusarium graminearum virus 1 (AAT07067); GaRV-L1, Gremmeniella

544 abietina RNA virus L1 (Q99AT3); GFLV, Grapevine fanleaf virus (P29149); HAV, Hepatitis

545 A virus (P26580); HRV-C, Human rotavirus C (Q91E95); LTSV, Lucerne transient streak

546 virus (Q83093); MAstV-1, Mink astrovirus 1 (AAO32082); PcV, Penicillium chrysogenum

547 virus (Q8JVC2); PEMV, Pea enation mosaic virus (P29154); PEV1, Phytophthora

548 endornavirus 1 (CAl47561); PiRV-1, Phytophthora infestans RNA virus 1 (FJ373316); PVY,

549 Potato virus Y (Q02963); RYMV, Rice yellow mottle virus (Q86519); ScV-L-A,

550 Saccharomyces cerevisiae virus L-A (Q87025); ScV-L-BC, Saccharomyces cerevisiae virus

551 L-BC (Q87027); SHV, Southampton virus (Q04544); SsRV1, Sphaeropsis sapinea RNA virus

5521 (Q9YXE6); TAstV-2, Turkey astrovirus 2 (AAF60952); TEV, Tobacco etch virus (P04517);

553 TMEV, Theiler's murine encephalomyelitis virus (P08544); and TVV, Trichomonas

554 vaginalis virus (Q90155). 
A
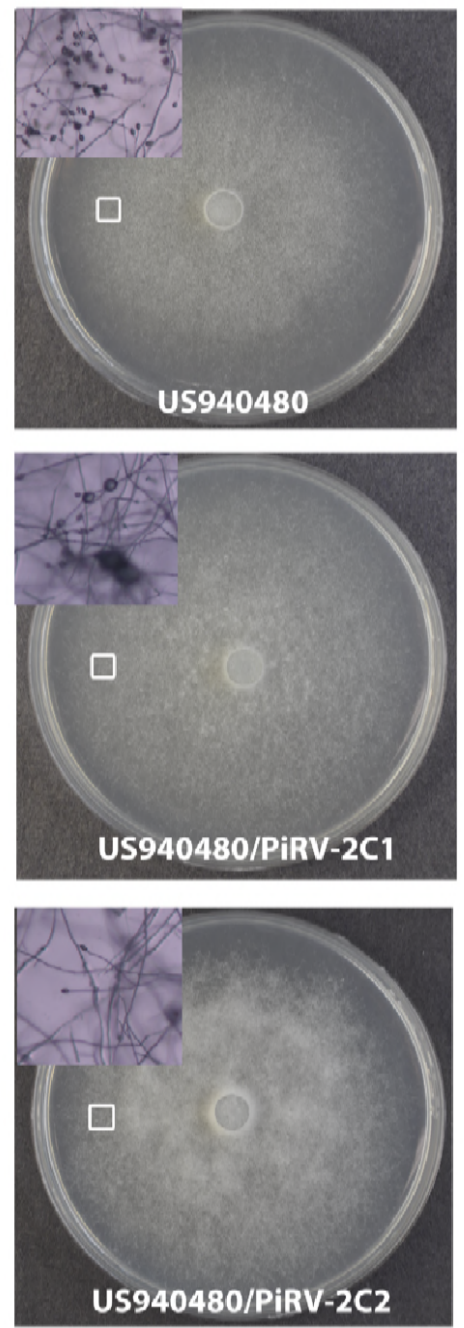

B

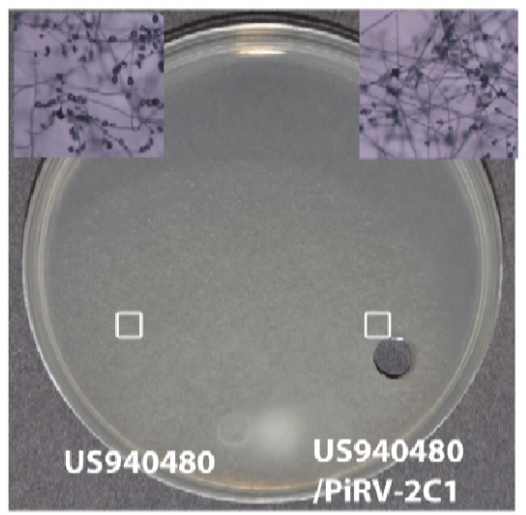

C

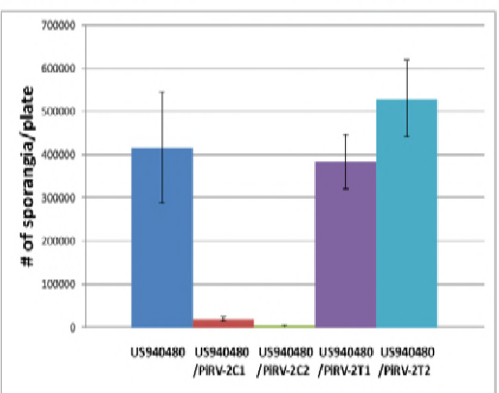

D

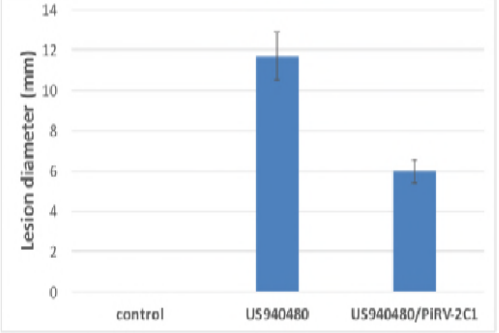

556 Fig. 3. PiRV-2 stimulates sporangia production in $P$. infestans and enhances its virulence.

557 (A) Colony morphology and sporulation (insets) of US940480, US940480/PiRV-2C1 and

558 US940480/PiRV-2C2. (B) Co-culturing of US940480 and US940480/PiRV-2C1 transmitted

559 the virus into the latter and restored its sporangia production. The white squares in $\mathbf{A}$

560 and B indicate the areas microscopically observed and photographed. An agar plug was

561 taken from the side of US940480/PiRV-2C1 to confirm virus transmission

562 (supplementary Figure S3). (C) Sporangia production in isogenic PiRV-2-containing and

563 PiRV-2-cured isolates. (D) Lesion size caused by isogenic PiRV-2-containing and PiRV-2-

564 cured isolates in detached leaf assay. Vertical bars, mean; and vertical lines, standard

565 error. Representative result from one of two experiments. 


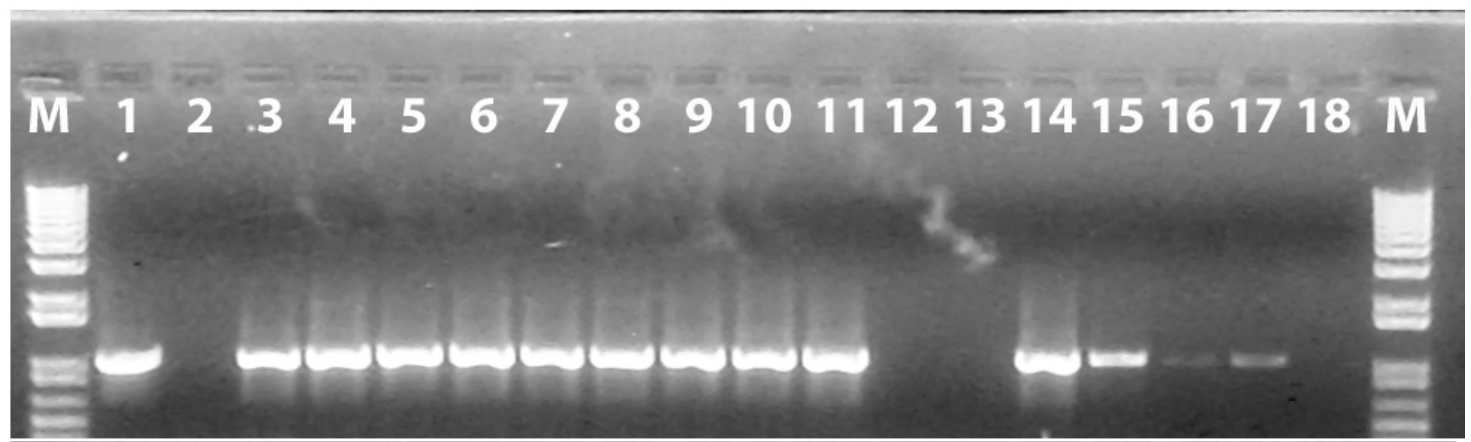

569 Fig. 4. RT-PCR detection of PiRV-2 in P. infestans collection. Upper panel, primer pair

570 P24/P27, lower panel, P16/P18. Lane designation: M, 1kb DNA ladder; 1, US940480; 2, 571 negative control; 3, US040009; 4, P8449; 5, P9017; 6, P9051; 7, P9200; 8, P9212; 9, 572 P9219; 10, P10107; 11, P10129; 12, US100001; 13, US100002; 14, US110001; 15, 573 P17707; 16, P17777; 17, P17783; and 18, P17785. 
bioRxiv preprint doi: https://doi.org/10.1101/383653; this version posted August 2, 2018. The copyright holder for this preprint (which was not certified by peer review) is the author/funder. This article is a US Government work. It is not subject to copyright under 17 USC 105 and is also made available for use under a CCO license.

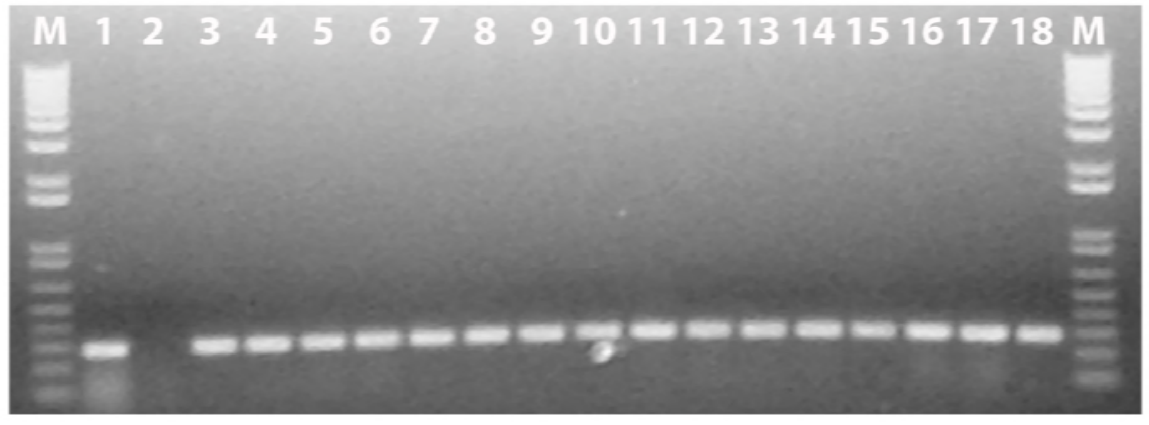

\section{192021222324252627282930313233}

577 Fig. 5. PiRV-2 transmission through sporangia. M, 1 kb DNA size marker, 1 and 19, 578 US940480; 2 and 20, negative controls; and 3-18 and 21-33, single sporangia cultures of 579 US940480. 


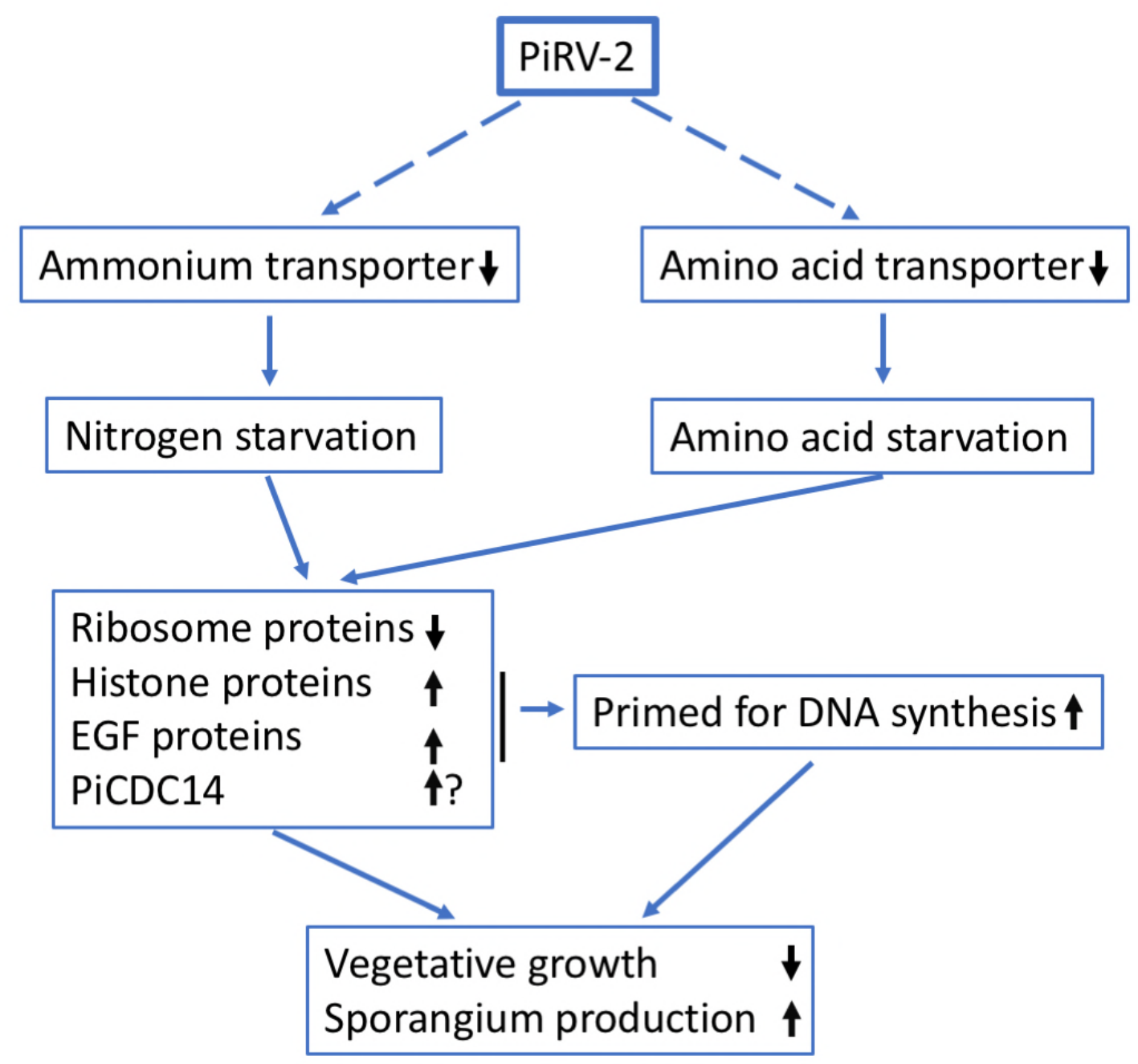

Fig.6. Potential molecular mechanism for PiRV-2-associated stimulation of sporangium 588 production in $P$. infestans. 


\section{References}

590 1. Bourke A. 'The visitation of god?' The potato and the great Irish famine. Dublin:

591 Lilliput Press Ltd; 1993. $x+230$ pp. p.

592 2. Haverkort AJ, Boonekamp PM, Hutten R, Jacobsen E, Lotz LAP, Kessel GJT, et al.

593 Societal costs of late blight in potato and prospects of durable resistance through

594 cisgenic modification. Potato Research. 2008;51(1):47-57. doi: 10.1007/s11540-008-

595 9089-y.

596 3. Guenthner JF, Michael KC, Nolte P. The economic impact of potato late blight on

597 US growers. Potato Research. 2001;44(2):121-5. doi: 10.1007/bf02410098.

598 4. USAblight, a national project on tomato \& potato late blight in the United States.

599 Available from: https://usablight.org/.

600 5. Sogin ML, Silberman JD. Evolution of the protists and protistan parasites from

601 the perspective of molecular systematics. International Journal for Parasitology.

602 1998;28:11-20.

603 6. Baldauf SL, Roger AJ, Wenk-Siefert I, Doolittle WF. A kingdom-level phylogeny of

604 eukaryotes based on combined protein data. Science. 2000;290(5493):972-7.

605 7. Cavalier-Smith T. Membrane heredity and early chloroplast evolution. Trends in

606 Plant Science. 2000;5(4):174-82.

607 8. Fry WE, Goodwin SB. Resurgence of the Irish potato famine fungus. Bioscience.

608 1997;47(6):363-71.

609 9. Fry WE, Goodwin SB. Recent migrations of Phytophthora infestans. In: Dowley LJ,

610 Bannon E, Cooke LR, Keane T, O'Sullivan E, editors. Phytophthora infestans: Boole Press,

611 Dublin, Ireland; 1995. p. 89-95.

612 10. Goodwin S, Cohen B, Fry W. Panglobal distribution of a single clonal lineage of

613 the Irish potato famine fungus. PNAS. 1994;91(24):11591-5.

614 11. Fry William E, Grunwald Niklaus J, Cooke David EL, McLeod A, Forbes Gregory A,

615 Cao K. Population genetics and population diversity of Phytophthora infestans. 2008.

616 doi:10.1002/9780470475898.ch7.

617 12. Fry WE, McGrath MT, Seaman A, Zitter TA, McLeod A, Danies G, et al. The 2009

618 late blight pandemic in the eastern United States - causes and results. Plant Dis.

619 2012;97(3):296-306. doi: 10.1094/PDIS-08-12-0791-FE.

620 13. Goodwin SB, Smart CD, Sandrock RW, Deahl KL, Punja ZK, Fry WE. Genetic

621 change within populations of Phytophthora infestans in the United States and Canada

622 during 1994 to 1996: role of migration and recombination. Phytopathology.

623 1998;88(9):939-49. Epub 2008/10/24. doi: 10.1094/phyto.1998.88.9.939.

624 14. Hu C-H, Perez FG, Donahoo R, McLeod A, Myers K, Ivors K, et al. Recent

625 genotypes of Phytophthora infestans in the eastern United States reveal clonal

626 populations and reappearance of mefenoxam sensitivity. Plant Dis. 2012;96(9):1323-30.

627 doi: 10.1094/PDIS-03-11-0156-RE.

628 15. Danies G, Small IM, Myers K, Childers R, Fry WE. Phenotypic Characterization of

629 recent clonal lineages of Phytophthora infestans in the United States. Plant Dis.

630 2013;97(7):873-81. doi: 10.1094/PDIS-07-12-0682-RE. 
631 16. Cai G, Myers K, Hillman BI, Fry WE. A novel virus of the late blight pathogen, 632 Phytophthora infestans, with two RNA segments and a supergroup 1 RNA-dependent 633 RNA polymerase. Virology. 2009;392(1):52-61.

634 17. Cai G, Myers K, Fry WE, Hillman BI. A member of the virus family Narnaviridae 635 from the plant pathogenic oomycete Phytophthora infestans. Arch Virol.

636 2012;157(1):165-9.

637 18. Cai G, Krychiw JF, Myers K, Fry WE, Hillman BI. A new virus from the plant 638 pathogenic oomycete Phytophthora infestans with an $8 \mathrm{~kb}$ dsRNA genome: The sixth 639 member of a proposed new virus genus. Virology. 2013;435(2):341-9.

640 19. Cai G, Hillman BI. Phytophthora Viruses. In: Said AG, editor. Adv Virus Res.

641 Volume 86: Academic Press; 2013. p. 327-50.

642 20. Kozak M. Point mutations define a sequence flanking the AUG initiator codon 643 that modulates translation by eukaryotic ribosomes. Cell. 1986;44(2):283-92. Epub 644 1986/01/31.

645 21. Lutcke HA, Chow KC, Mickel FS, Moss KA, Kern HF, Scheele GA. Selection of AUG 646 initiation codons differs in plants and animals. EMBO J. 1987;6(1):43-8.

647 22. Marchler-Bauer A, Bryant SH. CD-Search: protein domain annotations on the fly. 648 Nucleic Acids Research. 2004;32(suppl_2):W327-31.

649 23. Hulo N, Bairoch A, Bulliard V, Cerutti L, De Castro E, Langendijk-Genevaux PS, et 650 al. The PROSITE database. Nucl Acids Res. 2006;34(suppl_1):D227-30. doi:

$651 \quad 10.1093 /$ nar/gkj063.

652 24. Hofmann K, P. B, Falquet L, Bairoch A. The PROSITE database, its status in 1999. 653 Nucleic Acids Research. 1999;27:215-9.

654 25. Hansen JL, Long AM, Schultz SC. Structure of the RNA-dependent RNA

655 polymerase of poliovirus. Structure. 1997;5(8):1109-22.

656 26. Koonin EV, Dolja VV. Evolution and taxonomy of positive-strand RNA viruses:

657 Implications of comparative analysis of amino acid sequences. Critical Reviews in

658 Biochemistry and Molecular Biology. 1993;28(5):375-430.

659 27. Shwed PS, Dobos P, Cameron LA, Vakharia VN, Duncan R. Birnavirus VP1 proteins

660 form a distinct subgroup of RNA-dependent RNA polymerases lacking a GDD motif.

661 Virology. 2002;296(2):241-50.

662 28. Haas BJ, Kamoun S, Zody MC, Jiang RHY, Handsaker RE, Cano LM, et al. Genome

663 sequence and analysis of the Irish potato famine pathogen Phytophthora infestans.

664 Nature. 2009;461(7262):393-8.

665 29. Suzuki N, Ghabrial SA, Kim K-H, Pearson M, Marzano S-YL, Yaegashi H, et al. ICTV 666 virus taxonomy profile: Hypoviridae. J Gen Virol. 2018;99(5):615-6. doi:

667 doi:10.1099/jgv.0.001055.

668 30. Gahan PB. dsRNA genetic elements, concepts and applications in agriculture,

669 forestry and medicine S. T. Tavantzis (ed.), CRC Press, 284 pp., ISBN 0-849-22-05-7

670 (2002). Cell Biochem Funct. 2005;23(2):147-. doi: 10.1002/cbf.1123.

671 31. Pearson MN, Beever RE, Boine B, Arthur K. Mycoviruses of filamentous fungi and 672 their relevance to plant pathology. Mol Plant Pathol. 2009;10(1):115-28. 
673 32. Ghabrial SA, Caston JR, Jiang D, Nibert ML, Suzuki N. 50-plus years of fungal

674 viruses. Virology. 2015;479-480:356-68. Epub 2015/03/17. doi:

675 10.1016/j.virol.2015.02.034.

676 33. Nuss DL. Hypovirulence: Mycoviruses at the fungal-plant interface. Nature

677 Reviews Microbiology. 2005;3:632. doi: 10.1038/nrmicro1206.

678 34. Ahn IIP, Lee Y-H. A Viral Double-Stranded RNA Up Regulates the fungal virulence

679 of Nectria radicicola. Mol Plant-Microbe Interact. 2001;14(4):496-507. doi:

680 10.1094/MPMI.2001.14.4.496.

681 35. Okada R, Ichinose S, Takeshita K, Urayama S-i, Fukuhara T, Komatsu K, et al.

682 Molecular characterization of a novel mycovirus in Alternaria alternata manifesting two-

683 sided effects: Down-regulation of host growth and up-regulation of host plant

684 pathogenicity. Virology. 2018;519:23-32.

685 36. Schumann GL, D'Arcy CJ. Late blight of potato and tomato. The Plant Health

686 Instructor. 2000:DOI: 10.1094/PHI-I-2000-0724-01.

687 37. Goodwin SB, Sujkowski LS, Dyer AT, Fry BA and Fry WE 1995. Direct detection of

688 gene flow and probable sexual reproduction of Phytophthora infestans in northern

689 North America. Phytopathology 85: 473-479.

690 38. Kief DR, Warner JR. Coordinate control of syntheses of ribosomal ribonucleic acid

691 and ribosomal proteins during nutritional shift-up in Saccharomyces cerevisiae. Mol Cell

692 Biol. 1981;1(11):1007-15. doi: 10.1128/mcb.1.11.1007.

693 39. Nomura M. Organization of bacterial genes for ribosomal components: Studies

694 using novel approaches. Cell. 1974;9(4):633-44. doi: 10.1016/0092-8674(76)90127-6.

695 40. Shi YG, Tyler BM. Coordinate expression of ribosomal protein genes in

696 Neurospora crassa and identification of conserved upstream sequences. Nucleic Acids

697 Res. 1991;19(23):6511-7.

698 41. Pearson NJ, Haber JE. Changes in regulation of ribosomal protein synthesis

699 during vegetative growth and sporulation of Saccharomyces cerevisiae. J Bacteriol.

700 1980;143(3):1411-9.

701 42. Chu S, DeRisi J, Eisen M, Mulholland J, Botstein D, Brown PO, et al. The

702 transcriptional program of sporulation in budding yeast. Science. 1998;282(5389):699-

703 705. doi: 10.1126/science.282.5389.699.

704 43. Skromne I, Sanchez O, Aguirre J. Starvation stress modulates the expression of

705 the Aspergillus nidulans brlA regulatory gene. Microbiology. 1995;141 ( Pt 1):21-8. Epub

706 1995/01/01. doi: 10.1099/00221287-141-1-21.

707 44. Guignard R, Grange F, Turian G. Microcycle conidiation induced by partial

708 nitrogen deprivation in Neurospora crassa. Can J Microbiol. 1984;30(10):1210-5. doi:

709 10.1139/m84-192.

710 45. Warner JR, Gorenstein C. Yeast has a true stringent response. Nature.

711 1978;275:338. doi: 10.1038/275338a0.

712 46. Marian B, Wintersberger U. Histone synthesis during sporulation of yeast. FEBS

713 Lett. 1980;117(1):63-7.

714 47. Otto AM, Ulrich M-O, de Asua LJ. Epidermal growth factor initiates DNA

715 synthesis after a time-dependent sequence of regulatory events in Swiss 3T3 cells- 
716 interactions with hormones and growth factors. J Cell Physiol. 1981;108(2):145-53. doi:

$717 \quad 10.1002 / j c p .1041080205$.

718 48. Ng KW, Partridge NC, Niall M, Martin TJ. Stimulation of DNA synthesis by

719 epidermal growth factor in osteoblast-like cells. Calcif Tissue Int. 1983;35(1):624-8. doi:

720 10.1007/BF02405105.

721 49. Latijnhouwers M, Govers F. A Phytophthora infestans G-protein $\beta$ subunit Is

722 involved in sporangium formation. Eukaryot Cell. 2003;2(5):971-7. doi:

723 10.1128/ec.2.5.971-977.2003.

724 50. Ah Fong AM, Judelson HS. Cell cycle regulator Cdc14 is expressed during

725 sporulation but not hyphal growth in the fungus-like oomycete Phytophthora infestans.

726 Mol Microbiol. 2003;50(2):487-94. Epub 2003/11/18.

727 51. Judelson HS, Blanco FA. The spores of Phytophthora: weapons of the plant

728 destroyer. Nature Reviews Microbiology. 2005;3:47. doi: 10.1038/nrmicro1064.

729 52. Morris TJ, Dodds JA. Isolation and analysis of double-stranded RNA from virus-

730 infected plant and fungal tissue. Phytopathology. 1979;69:854-8.

731 53. Tooley PW, Hewings AD, Falkenstein KF. Detection of double-stranded RNA in

732 Phytophthora infestans. Phytopathology. 1989;79(4):470-4.

733 54. Attoui H, Billoir F, Cantaloube JF, Biagini P, de Micco P, de Lamballerie X.

734 Strategies for the sequence determination of viral dsRNA genomes. Journal of

735 Virological Methods. 2000;89(1-2):147-58.

736 55. Lambden PR, Cooke SJ, Caul EO, Clarke IN. Cloning of noncultivatable human

737 rotavirus by single primer amplification. J Virol. 1992;66(3):1817-22.

738 56. Petersen TN, Brunak S, von Heijne G, Nielsen H. SignalP 4.0: discriminating signal

739 peptides from transmembrane regions. Nat Meth. 2011;8(10):785-6.

740 57. Krogh A, Larsson B, von Heijne G, Sonnhammer ELL. Predicting transmembrane

741 protein topology with a hidden markov model: application to complete genomes1. J Mol

742 Biol. 2001;305(3):567-80.

743 58. Tamura K, Stecher G, Peterson D, Filipski A, Kumar S. MEGA6: Molecular

744 Evolutionary Genetics Analysis Version 6.0. Mol Biol Evol. 2013;30(12):2725-9. doi:

$745 \quad 10.1093 / \mathrm{molbev} / \mathrm{mst} 197$.

746 59. Andrews S. FastQC, a quality control tool for high throughput sequence data.

747 https://wwwbioinformaticsbabrahamacuk/projects/fastqc/ [Internet]. 2010.

748 60. Hannon Lab. FASTX-Toolkit. http://hannonlabcshledu/fastx toolkit/indexhtml

$749 \quad$ [Internet]. 2010.

750 61. Trapnell C, Pachter L, Salzberg SL. TopHat: discovering splice junctions with RNA-

751 Seq. Bioinformatics. 2009;25(9):1105-11. doi: 10.1093/bioinformatics/btp120.

752 62. Langmead B, Salzberg SL. Fast gapped-read alignment with Bowtie 2. Nat Meth.

753 2012;9(4):357-9. doi: 10.1038/nmeth.1923

754 63. Anders S, Pyl PT, Huber W. HTSeq-a Python framework to work with high-

755 throughput sequencing data. Bioinformatics. 2015;31(2):166-9. doi:

756 10.1093/bioinformatics/btu638.

757 64. Love MI, Huber W, Anders S. Moderated estimation of fold change and

758 dispersion for RNA-seq data with DESeq2. Genome Biol. 2014;15(12):550. doi:

759 10.1186/s13059-014-0550-8. 
bioRxiv preprint doi: https://doi org/10.1101/383653; this version posted August 2, 2018. The copyright holder for this preprint (which was not certified by peer review) is the author/funder. This article is a US Government work. It is not subject to copyright under 17 USC 105 and is also made available for use under a CCO license.

760 65. Huang DW, Sherman BT, Lempicki RA. Systematic and integrative analysis of 761 large gene lists using DAVID bioinformatics resources. Nat Protoc. 2008;4:44. doi:

762 10.1038/nprot.2008.211

763

764 


\section{Supporting information}

\section{Supplementary Figures}

767 Table S1. Isolates of Phytophthora infestans used in the current study

768 Table S2. Anotation of primers and adapters used in the current study

769 Table S3. Impact of PiRV-2 on host gene expression

770 Table S4. Effect of PiRV-2 on expression of histone protein genes and histone modifying 771 enzyme genes

772 Table S5. Effect of PiRV-2 on expression of genes in KEGG ribosome pathway

773 Table S6. Transmembrane proteins down-regulated by PiRV2

774 Table S7. Effect of PiRV-2 on the expression of ammonium transpoter and amino acid 775 permease genes 